\title{
Biochemical characterization of an alcohol dehydrogenase from Pyrus ussuriensis 'Nanguoli'
}

\author{
Qin Gaihua ${ }^{1,2}$, Liu Chunyan ${ }^{1,2}$, Gao Zhenghuii ${ }^{1,2}$, Li Jiyu ${ }^{1,2}$, Qi Yongjie ${ }^{1,2}$, Pan Haifa $^{1}$, Yi Xingkai ${ }^{1}$ and \\ $\mathrm{Xu}$ Yiliu ${ }^{1,2}$ \\ ${ }^{1}$ Key Laboratory of Horticultural Crop Genetic Improvement and Eco-physiology of Anhui Province, Institute of Horticulture \\ Research, Anhui Academy of Agricultural Sciences, Hefei, China \\ ${ }^{2}$ Key Laboratory of Fruit Quality and Developmental Biology, Anhui Academy of Agricultural Sciences, Hefei, China
}

\begin{abstract}
Summary
Alcohol dehydrogenase (ADH) is an oxidation-reduction enzyme that catalyzes interconversion of alcohols and aldehydes and provides a substrate for the formation of esters. Although candidate genes encoding ADHs have been identified in pear fruits, the characterization of their encoded proteins remains unknown. In this study, an $A D H$ was cloned from a cDNA of Pyrus ussuriensis 'Nanguoli' (PUADH2) and was expressed in Escherichia coli. The recombinant PuADH2 protein was purified and subject to biochemical characterization. PuADH2 was more likely an oxidative enzyme independent of substrate specificity. During oxidation reaction, no preference for coenzyme factors (NAD ${ }^{+}$and $\mathrm{NADP}^{+}$) for PuADH2 oxidase activity. In addition, the oxidation activity of $P U A D H 2$ slightly increased with high $\mathrm{K}^{+}$concentrations, but significantly decreased with $\mathrm{Zn}^{2+}$. These results provide insights into $\mathrm{PUADH} 2$ as an oxidase and promote future study on the synthesis and regulation of aromatic compounds in pear fruits.
\end{abstract}

Keywords

recombinant protein, oxidase, fruit aroma, enzyme activity

\section{Introduction}

Fruit aroma is determined by a complex mixture of volatile compounds. Pear fruits have hundreds of aromatic compounds and volatile esters are the key compounds contributing to the development of their characteristic aroma (Qin et al., 2012). A previous report showed that ester production in pear fruits was limited to the concentration of precursors (Qin et al., 2017). Alcohol dehydrogenases (ADHs; EC 1.1.1.1) are key enzymes that catalyze the interconversion between aldehydes and the corresponding alcohols and participate in the process of ester-based aroma formation.

To date, all plants were found to carry multiple $A D H$ genes, except for a few Arabidopsis species. Several $A D H$ genes have been characterized in plants, including melons (Manriquez et al., 2006), apricots (González-Agüero et al., 2009), peaches (Zhang et al., 2010), and tomatoes (Longhurst et al., 1994). In melons, expression of Candida maltose $(\mathrm{Cm}) \mathrm{ADH} 1$ and $\mathrm{CmADH} 2$ showed increasing patterns during ripening (Manriquez et al., 2006). In contrast, PpADH1, PpADH2, and PpADH3 in peaches (Prunus persica) were down-regulated during ripening (Zhang et al., 2010).

\section{Significance of this study}

What is already known on this subject?

- Alcohol dehydrogenases are key enzymes that catalyze the interconversion between aldehydes and the corresponding alcohols and participate in the process of ester-based aroma formation.

What are the new findings?

- Biochemical analysis suggested that PuADH2 be an oxidase. Its activity was enhanced by high $\mathrm{K}^{+}$concentrations, but significantly decreased with $\mathrm{Zn}^{2+}$.

What is the expected impact on horticulture?

- Biochemical characterization of an alcohol dehydrogenase will help to understand its role in ester-volatile synthesis in pear fruits and promote future study on the synthesis and regulation of aromatic compounds in pear fruits.

In pears (Pyrus bretschneideri), the total activity of eight PbrADHs and volatile ester production were strongly associated with increased PbrADH6 transcription (Qin et al., 2017). However, the expression pattern of $\mathrm{PbrADH} 2$ was opposite to that of PbrADH6, whose biochemical characteristics require further study.

Pyrus ussuriensis 'Nanguoli' has pleasant flavor produced by volatile esters, attractive color and exquisite flesh, therefore the cultivar was spreading in Northern China. Compared to Pyrus ussuriensis 'Nanguoli', P. bretschneideri 'Dangshansuli' produces many straight-chain alcohols and aldehydes, generating fruits a "green" flavor note. In this study, we cloned an $A D H$ gene, $P U A D H 2$, from a cDNA of ripened 'Nanguoli' fruits and expressed PUADH2 in Escherichia coli BL21. The recombinant PuADH2 protein was characterized biochemically, including oxidant/reduction activity and effects of cofactors and metal ions, in order to understand its role in ester-volatile synthesis in pear fruits.

\section{Materials and methods}

\section{Materials}

Ripen pear (Pyrus ussuriensis 'Nanguoli') fruits were obtained from the orchard at Anhui Academy of Agricultural Sciences. All enzymes used in recombinant plasmid construction were bought from TaKaRa (Dalian, China). Polymerase chain reaction (PCR) product purification and plasmid miniprep kits were purchased from Beijing TransGen Biotech Co., 
Ltd. (Beijing, China). The pET28a was bought from Novagen (Darmstadt, Germany).

\section{Sequence analysis of $\mathrm{PuADH2}$}

Multiple sequence alignment of PuADH2 and its homologs were performed with Clustal X (Thompson et al., 1997). A phylogenetic tree was constructed by using the Neighborjoining (NJ) method (Saitou and Nei, 1987) of MEGA 5 software (www.megasoftware.net/index.html). Threedimensional (3D) structure of $\mathrm{PuADH} 2$ was predicted following Biasini (2014).

\section{Amplification and cloning of $P U A D H 2$}

Total RNA from the fruit of $P$. ussuriensis 'Nanguoli' was extracted according to Asif et al. (2000), which was then used to synthesize the cDNAs using the PrimeScript ${ }^{\mathrm{TM}}$ RT reagent kit (TaKaRa). A full-length cDNA fragment was amplified with the sequences $P U A D H 2$-specific-forward: 5'-CGGGATCCATGTCTAATACTGCTGGTCAGGT-3' and PuADH2specific-reverse: $5^{\prime}$-CCGCTCGAGTTCCTCCATGTGGATTATGC AACG-3'. The thermal cycle program was: $3 \mathrm{~min}, 95^{\circ} \mathrm{C}$; 30 cycles at $\left(95^{\circ} \mathrm{C}, 30 \mathrm{~s} ; 60^{\circ} \mathrm{C}, 45 \mathrm{~s} ; 72^{\circ} \mathrm{C}, 60 \mathrm{~s}\right)$; and $72^{\circ} \mathrm{C}$, $10 \mathrm{~min}$. The PUADH2 cDNA was gel purified and sub-cloned into the pMD19-T vector and verified by sequencing (Sangon Biotech).

\section{Expression and purification of $\mathrm{PUADH} 2$}

The coding sequence (excluding the stop codon) of PuADH2 was cloned into the pET28a vector with His6-tag, the recombinant PuADH2 was transformed into E. coli BL21 (DE3) cells. Protein expression was induced with isopropyl $\beta$-D-1-thiogalactopyranoside (IPTG, $0.1 \mathrm{mM}$ ) at $37^{\circ} \mathrm{C}$ for $4 \mathrm{~h}$. The cell cultures were harvested by centrifugation and re-suspended in $50 \mathrm{~mL}$ buffer $(300 \mathrm{mM} \mathrm{NaCl}, 5 \%$ glycerol, $1 \mathrm{mM}$ PMSF, $20 \mathrm{mM}$ Tris- $\mathrm{HCl}, \mathrm{pH}$ 8.0, and $5 \mathrm{mM}$ imidazole) and sonicated for $30 \mathrm{~min}(300 \mathrm{~W}$, with a 6-s pulse and an 8-s pause). The lysate was centrifuged and the pellet was washed using Tris-HCl (20 mM, pH 8.0) twice and re-suspended in $5 \mathrm{~mL}$ of solubilizing buffer $(20 \mathrm{mM}$ Tris buffer, pH 8.0, $7 \mathrm{M}$ urea) to achieve complete solubilization. The supernatant was obtained by centrifuging and then was transferred to $50 \mathrm{~mL}$ refolding buffer (20 mM Tris buffer, $\mathrm{pH}$ 8.0, $10 \%(\mathrm{w} / \mathrm{v})$ sucrose, $2 \mathrm{mM} \mathrm{CaCl}_{2}$ ).

Following the method of Kumar et al. (2015) with modifications, the refolded protein sample was purified by chromatograph passing through a Ni-NTA affinity column containing $5 \mathrm{~mL}$ of nickel beads (Novagen). After the loading, the column was washed with $50 \mathrm{~mL}$ wash buffer in step-wise increments as follows: buffer $1(150 \mathrm{mM} \mathrm{NaCl}$ and $20 \mathrm{mM}$ imidazole); buffer 2 (150 $\mathrm{mM} \mathrm{NaCl}$ and $40 \mathrm{mM}$ imidazole); buffer 3 (150 $\mathrm{mM} \mathrm{NaCl}$ and $60 \mathrm{mM}$ imidazole); and buffer 4 (150 mM NaCl and $80 \mathrm{mM}$ imidazole). His-tagged PuADH2 was eluted then with $10 \mathrm{~mL}$ of wash buffer containing (150 mM NaCl, $400 \mathrm{mM}$ imidazole). The elution was concentrated to $2 \mathrm{~mL}$ in a $10 \mathrm{~K}$ Ultracel centrifugation filter (Merck Millipore, Billerica, MA, U.S.A.) and diluted with a low-salt buffer containing $20 \mathrm{mM}$ Tris- $\mathrm{HCl}$ (pH 8.0).

Protein purity was verified by $10 \%$ SDS-PAGE, and the proteins were transferred to a nitrocellulose (NC) membrane. The NC membrane was incubated for $1 \mathrm{~h}$ with the rabbit anti-His monoclonal antibody. After washing with $1 \times$ PBST, the NC membrane was incubated with horseradish peroxidase-labeled goat anti-rabbit secondary antibody for $1 \mathrm{~h}$. The NC membrane was then washed five times with $1 \times$ PBST as described before exposure.

\section{Measurement of enzyme activity}

The reduction and oxidation activities of PuADH2 were quantified by spectrophotometry following the method of Molina et al. (1987). Activity of PuADH2 reductase was measured with $2.4 \mathrm{mM} \mathrm{NADH}, 33 \mathrm{mM}$ acetaldehyde, $50 \mathrm{mM}$ PBS (pH 7.0), and 12 15 $\mu \mathrm{g}$ PuADH2. Activity of PuADH2 dehydrogenase was assayed in the presence of $2.4 \mathrm{mM} \mathrm{NADH}$, $33 \mathrm{mM}$ ethanol, $50 \mathrm{mM}$ PBS (pH 7.0), and between 12 15 $\mu \mathrm{g}$ PuADH2. PuADH2 was replaced with $50 \mathrm{mM}$ PBS (pH 7.0) for the control. The OD was detected by spectrophotometry at $340 \mathrm{~nm}$ every $15 \mathrm{~s}$ until no change in the OD value was observed. All measurements were performed in triplicate. The results were expressed as specific activity (U mg-1 protein).

\section{Cofactor specificity}

To evaluate cofactor preference, $\mathrm{NAD}^{+}$and $\mathrm{NADP}^{+}$were used to investigate the oxidation activity of $\mathrm{PUADH} 2$ in a reaction mixture containing $2.4 \mathrm{mM} \mathrm{NAD}^{+}$or $\mathrm{NADP}^{+}, 33 \mathrm{mM}$ ethanol, 50 mM PBS (pH 7.0), 12 15 $\mu \mathrm{g}$ PuADH2. Each assay was performed in triplicate.

\section{Substrate specificity}

Substrate specificity was studied with phenylacetaldehyde, ethanol, or hexanal as the substrate in reduction reactions. The oxidation activity of $P U A D H 2$ was further analyzed using hexanol as a substrate.

\section{Effect of metal ions on enzyme activity}

PuADH2 is soluble in PBS, whereas it is insoluble in PBS with high concentrations of $\mathrm{Ca}^{2+}, \mathrm{Mg}^{2+}$, or $\mathrm{Zn}^{2+}$. To address this issue, $3 \mathrm{mg} P U A D H 2$ was dialyzed in MES buffer $(50 \mathrm{mM}$, $\mathrm{pH}$ 6.0) overnight for buffer exchange at $4^{\circ} \mathrm{C}$. Effect of metal ions on PUADH2 activity was conducted with reaction solutions with $33 \mathrm{mM}$ ethanol, $2.4 \mathrm{mM} \mathrm{NAD}^{+}$and different concentrations of $\mathrm{Ca}^{2+}, \mathrm{Mg}^{2+}, \mathrm{K}^{+}$or $\mathrm{Zn}^{2+}$. The final concentrations of $\mathrm{Ca}^{2+}$ and $\mathrm{K}^{+}$were $0 \mu \mathrm{M}, 10 \mu \mathrm{M}, 25 \mu \mathrm{M}$, and $50 \mu \mathrm{M}$, whereas the final concentrations of $\mathrm{Mg}^{2+}$ and $\mathrm{Zn}^{2+}$ were $0 \mu \mathrm{M}, 1 \mu \mathrm{M}$, $2 \mu \mathrm{M}$, and $5 \mu \mathrm{M}$ (Elleuche et al., 2014), respectively. The concentration of PuADH2 was 7 8 $\mu$ g.

\section{Statistical analysis}

Statistical analysis was done through SPSS software (IBM Corp., Armonk, NY, U.S.A.). The $t$-test $(p<0.05)$ was used for two groups and Kruskal-Wallis one-way ANOVA $(p<0.05)$ was used for more than two groups to test the significant differences.

\section{Results}

\section{PuADH2 sequence analysis and phylogeny}

PuADH2 (GenBank ID: KU507377) has an open reading frame of 1,140 bp and encodes a 380-amino acid protein with a predicted molecular weight of $46 \mathrm{kDa}$. An Interproscan (Philip Jones et al., 2014) of the sequence revealed that these sequences contained motifs characteristic of ADH. The alignment of the ADHs shared two major conserved domains, including a GroES-like domain (between residues 35-164) and a zinc-binding domain (between residues 206340) (Figure 1). Gene Ontology prediction indicated that $\mathrm{PuADH} 2$ may involve in oxidation-reduction processes (GO: 0055114) with zinc-binding (GO: 0008270) and oxidoreductase (GO: 0016491) activities. Additionally, there were high levels of sequence identity (84.7-96.8\%) between PuADH2 and zinc-medium-chain ADHs subfamily members of other plants (Figure 1). The phylogenetic tree revealed that the 


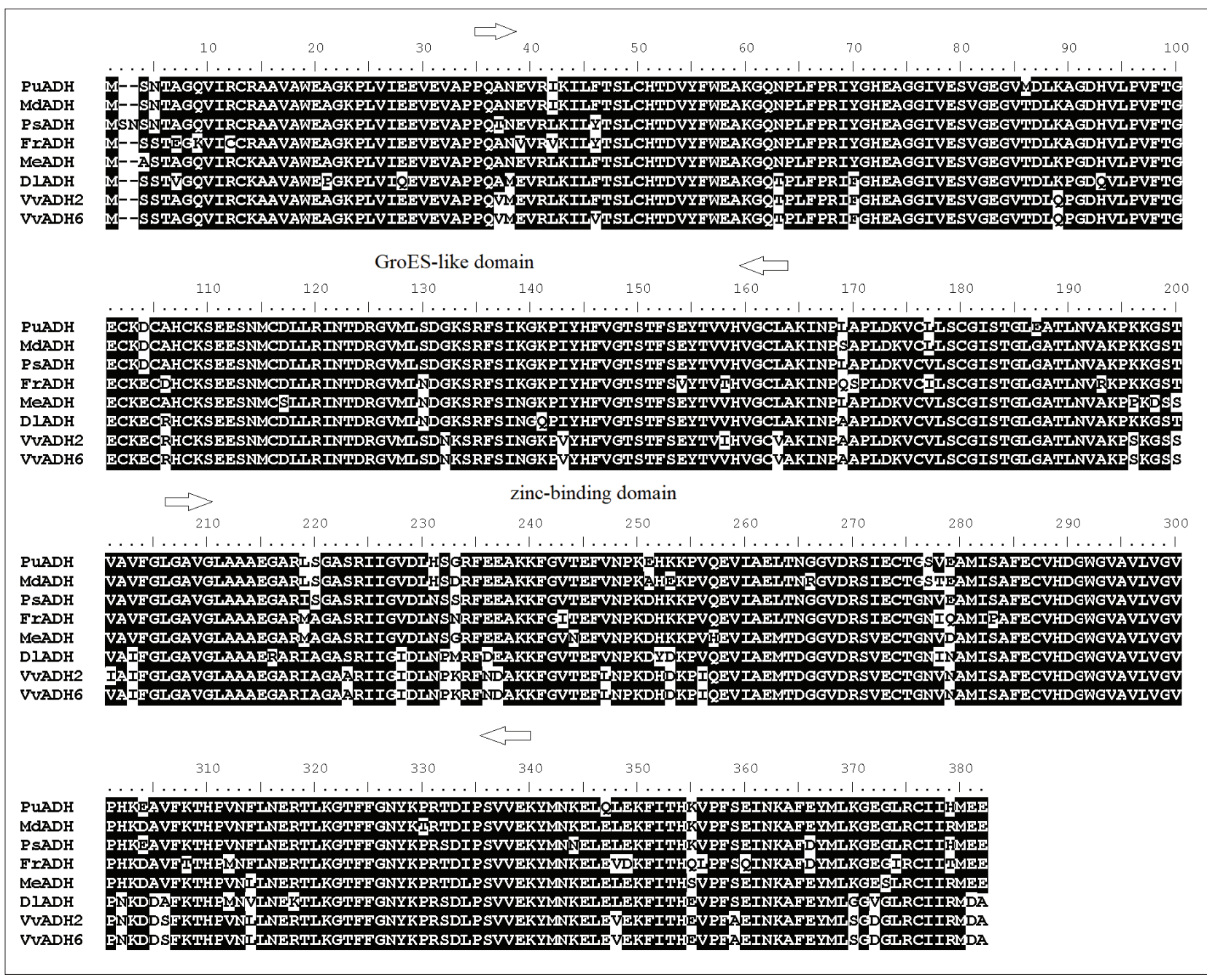

FIGURE 1. Amino acid sequences alignment of Pyrus ussuriensis PuADH2 with closely related full length sequences of Malus domestica MdADH (CAA88271.1), Prunus avium PsADH (XP_021833624.1), Fragaria $\times$ ananassa FrADH (CAA33613.1), Manihot esculenta, MeADH (XP_021622038.1), Dimocarpus longan DlADH (ABF61806.1), Vitis vinifera VvADH2 (NP_001268083.1), and V. vinifera, VvADH6 (AAF44335.1). Black shading indicates similar residues. Functional domains were predicted using Interproscan. A GroES-like domain and a structural zinc-binding domain were identified.

$15 \mathrm{ADH}$ family members were divided into two groups, with the closest neighbor of $P U A D H 2$ being $M d A D H$ from Malus domestica (Figure 2). As was predicted, the 3D structure of $\mathrm{PuADH} 2$ existed as a homodimer containing $\mathrm{Zn}^{2+}$ binding sites (Figure 3).

\section{PUADH2 gene expression}

Upon induction of PuADH gene expression, $\mathrm{pE}$ T28a-transformed BL21 cells produced a 46-kDa PuADH2 protein (Figure 4A). The Western blot analysis revealed two specific bands of approximately $46 \mathrm{kDa}$ and $92 \mathrm{kDa}$ molecular masses (Figure 4B), representing the monomer and homodimer forms of the protein. The PuADH2 protein was mainly expressed in the form of inclusion bodies and the bioactive $\mathrm{PuADH} 2$ protein was obtained through solubilization and refolding procedures as described in methods.

\section{PUADH2 oxidation/reduction activity}

Purified PUADH2 was tested for reductase or oxidase activity. Although a higher reduction activity was shown by PuADH2 with acetaldehyde as a substrate comparing to its oxidation activity with ethanol serving as a substrate; however, its overall oxidation activity was higher than its reduction

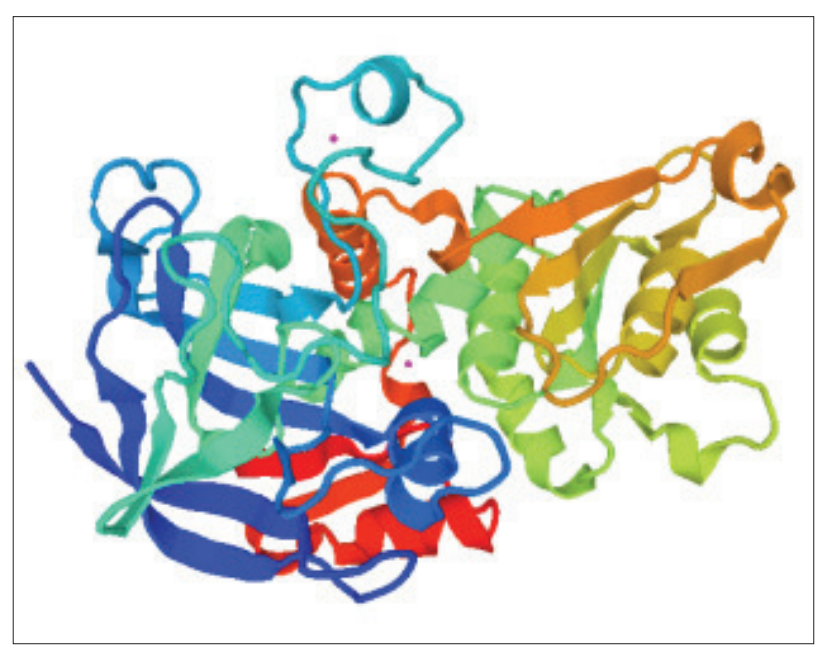

FIGURE 3. 3D structure of PuADH2 predicted according to its translated protein sequence. $\mathrm{Zn}^{2+}$ ions are shown in pink.

activity (Figure 5A). This was confirmed by using hexanol as a substrate, suggesting that $P U A D H 2$ acts preferentially as an oxidase (Figure 5B). 


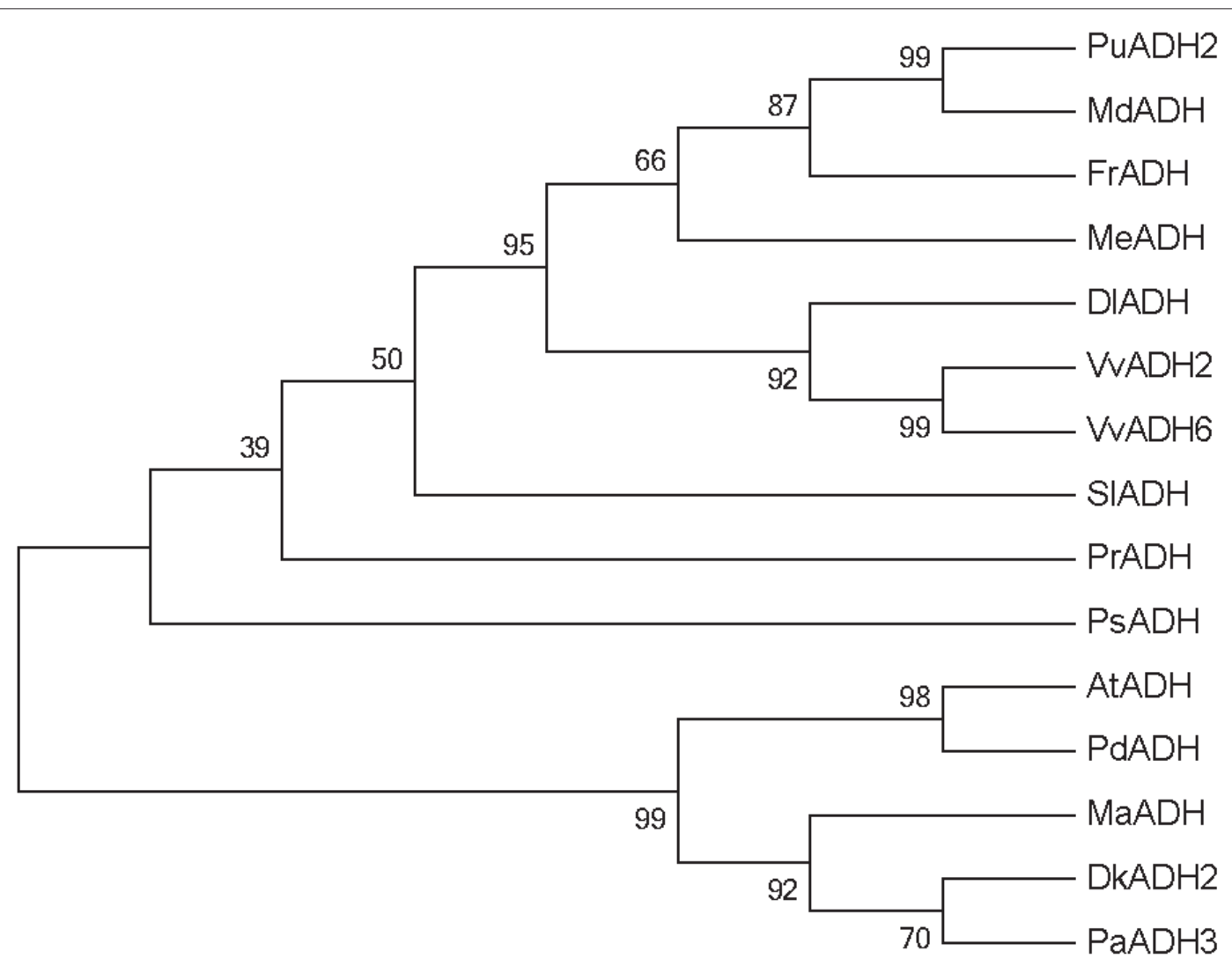

FIGURE 2. Phylogenetic trees for PuADH2 with other ADH sequences. Numbers above the branches indicate bootstrap values (1,000 replicates). Sequences are from Malus domestica MdADH (CAA88271.1); Fragaria $\times$ ananassa FrADH (CAA33613.1), Manihot esculenta MeADH (XP_021622038.1), Dimocarpus longan DIADH (ABF61806.1), Vitis vinifera VvADH2 (NP_001268083.1), V. vinifera VvADH6 (AAF44335.1), Solanum lycopersicum SlADH (AAB33481.2), Prunus armeniaca PrADH (ABZ79222.1), Prunus avium PsADH (XP_021833624.1), Arabidopsis thaliana AtADH (AAS45601.2), Prunus dulcis $\times$ Prunus persica PdADH (AEF30408.2), Musa acuminata colla MaADH (AIU98498.1), Diospyros kaki DkADH2 (AGA15794.1), Persea americana PaADH3(ALR87070.1).

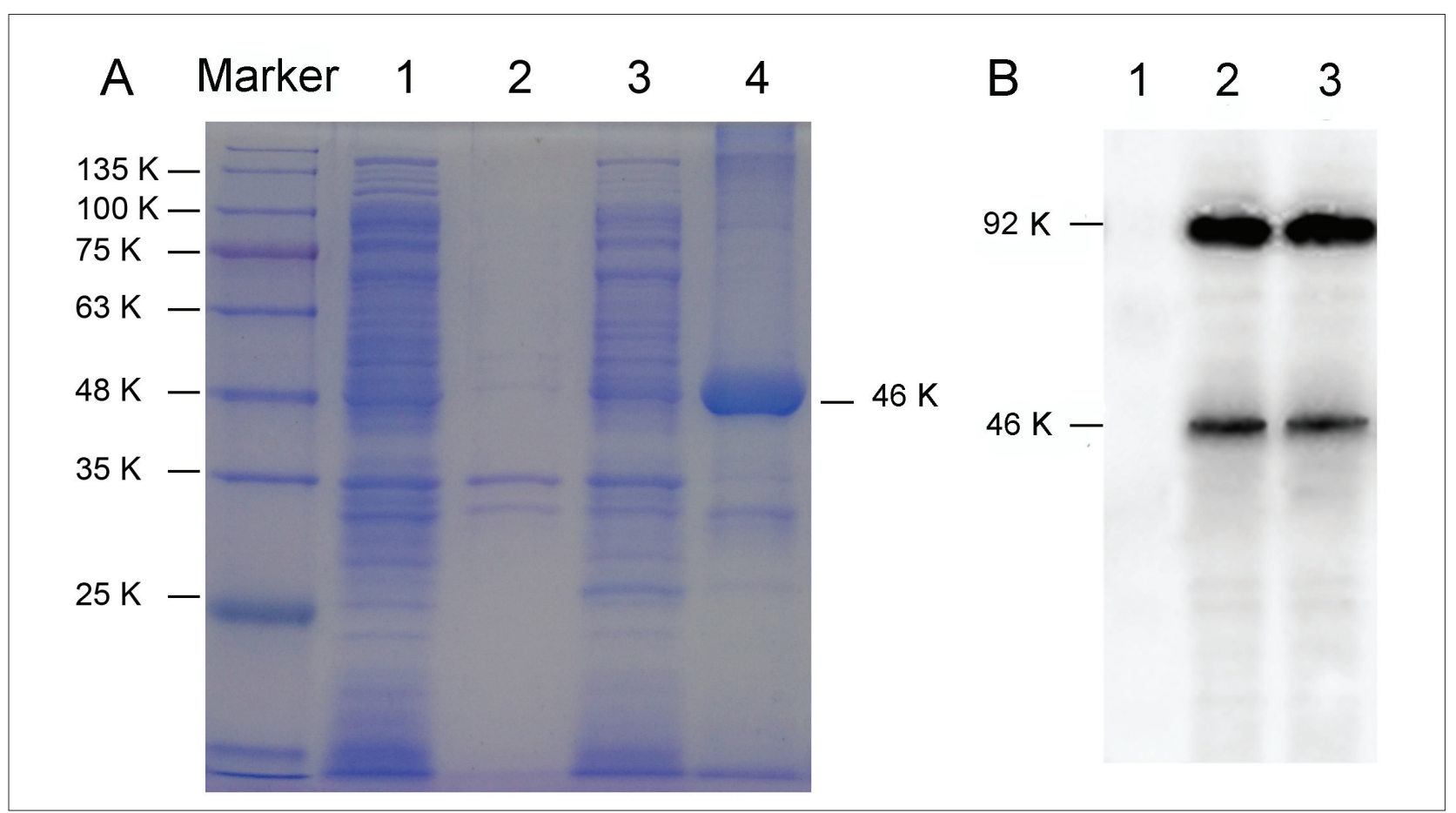

FIGURE 4. SDS-PAGE and western blot of purified PuADH2. (A) M, marker; lane 1, supernatant before induction; lane 2, precipitant before induction; lane 3, supernatant after induction; and lane 4, precipitant after induction. (B) The proteins separated on non-reducing SDS-PAGE gels were transferred to NC membranes for western blot analysis. Band sizes were $\sim 92 \mathrm{kDa}$ and $\sim 46 \mathrm{kDa}$, respectively. Lanes 2 and 3 were duplicates. 
When phenylacetaldehyde, ethanal, hexanal, and hexanol were used as the substrates, PuADH2 had the higher oxidase activity when hexanol was used as a substrate, indicating its preferential oxidase activity was not substrate-specific (Figure 5B).

\section{The effect of cofactors on PuADH2 activity}

Upon addition of $\mathrm{NADP}^{+}$, reaction plateaued after $3 \mathrm{~min}$, which was sooner than $\mathrm{NAD}^{+}$addition. The final enzyme activity of PuADH2 in the presence of $\mathrm{NADP}^{+}(3,396 \pm 68.5$ $\left.\mathrm{U} \mathrm{mg}^{-1}\right)$ was lower than that with $\mathrm{NAD}^{+}\left(4,237 \pm 45.7 \mathrm{U} \mathrm{mg}^{-1}\right)$ (Figure $5 \mathrm{C}$ ), but there is no significant difference between the two groups, suggesting no preference for coenzyme factors for PUADH2 oxidase activity.

\section{The effect of some metal ions on PuADH2 oxidase activity}

Minimal effects on PUADH2 oxidase activity were observed when $10-50 \mu \mathrm{M} \mathrm{Ca}^{2+}$ or $10-25 \mu \mathrm{M} \mathrm{K}^{+}$was present in the reaction. However, addition of $50 \mu \mathrm{M} \mathrm{K}^{+}$significantly enhanced the enzyme activity $(p<0.05)$ (Figure 6A). Additionally, the $\mathrm{PuADH} 2$ oxidase activity was slightly elevated by $\mathrm{Mg}^{2+}$, but significantly suppressed by $\mathrm{Zn}^{2+}(p<0.05)$ at all tested concentrations (Figure 6B).

\section{Discussion}

ADH genes can catalyze the reciprocal transformation between alcohols and aldehydes and are participates in ester-aroma formation, in additional ADH participate in plant development and stress responses (Singh et al., 2018). Eight $A D H$ genes were identified in Chinese white pear, and the association between $A D H$ activity and aromatic compounds was determined (Qin et al., 2017). Here, we cloned the PuADH2 gene from P. ussuriensis determined 'Nanguoli', which reportedly has a "fruity" aroma (Wu et al., 2013). We also expressed the gene in E. coli and isolated and biochemically characterized the recombinant protein PuADH2. The PuADH2 protein is a member of the zinc-medium-chain ADH subfamily (Chase, 1999), which has multiple genes involved in the production of aromatic compounds during fruit ripening. Transgenic tomato fruit expressing MiADH1 gene showed a change in the level of several alcohols and aldehydes related to flavor (Singh et al., 2018). Lipoxygenase
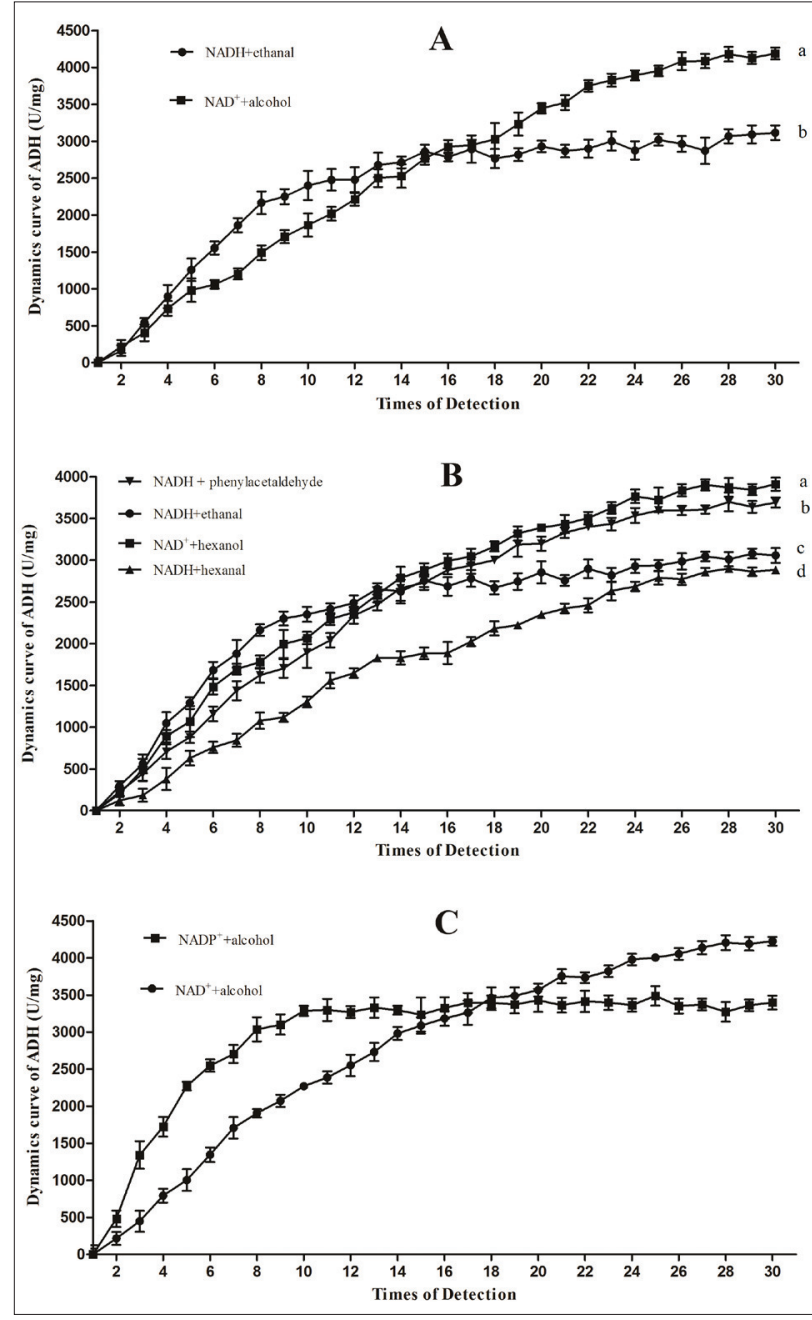

FIGURE 5. The X-axis represents detection frequency, and the Y-axis represents the activity units at each detection time. All values are the mean \pm standard error of three replicates. (A) PuADH2 oxidation-reduction reactions. Different lowercase letters indicate significant differences. (B) PuADH2 oxidation-reduction reactions with different substrates. Different lowercase letters indicate significant differences. (C) PuADH2 oxidation-reduction reactions with different cofactors.
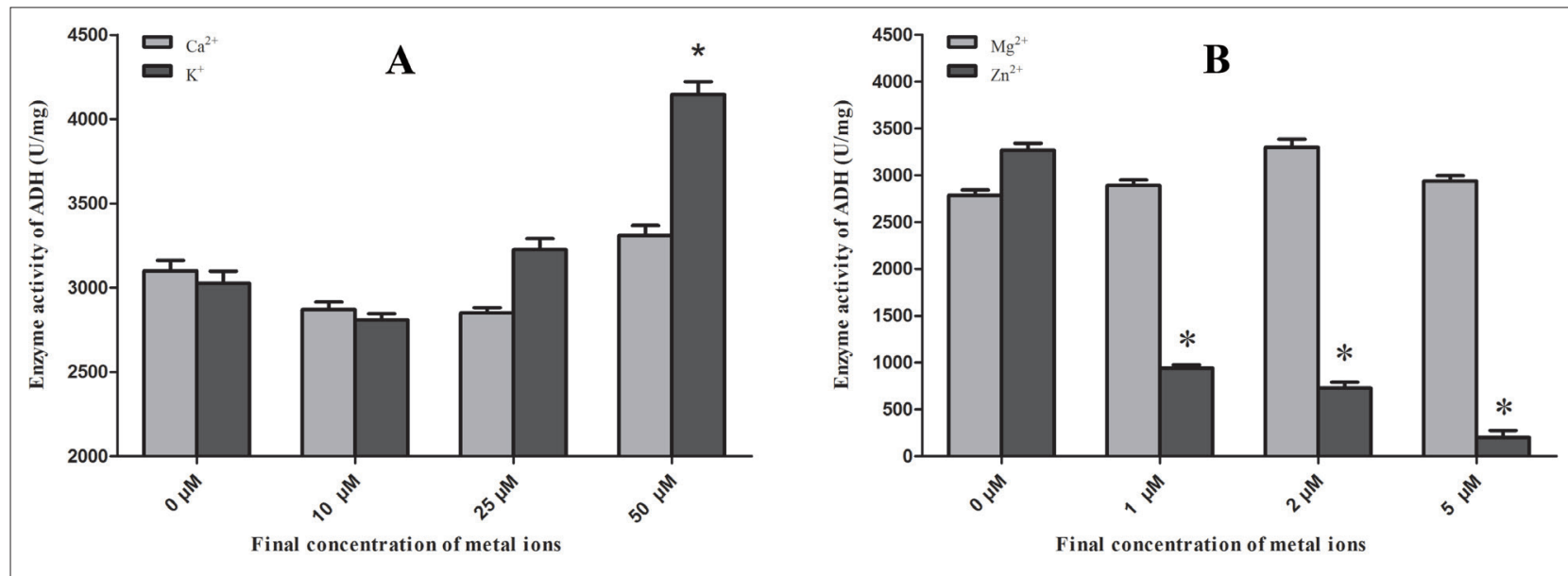

FIGURE 6. (A) PuADH2 oxidase activity in the presence of different concentrations of $\mathrm{Ca}^{2+}$ and $\mathrm{K}^{+}$. The $\mathrm{X}$-axis represents detection frequency, and the Y-axis represents oxidase activity. All values are the mean \pm standard error of three replicates. (B) PuADH2 oxidase activity in the presence of different concentrations of $\mathrm{Mg}^{2+}$ and $\mathrm{Zn}^{2+}$. The $\mathrm{X}$-axis represents detection frequency, and the Y-axis represents oxidase activity. All values are the mean \pm standard error of three replicates. Asterisks indicate the significant differences in the oxidase activity compared to $0 \mu \mathrm{M}$ metal ion treatment condition in each group. 
pathway is confirmed as an important biosynthetic mechanism for the generation of free and glycosylated volatile organic compounds during tamarillo ripening and this biosynthesis pathway is highly correlated with the activities of ADHs (Chen et al., 2020). A medium-chain protein encoding CmADH1 was identified in melon and this gene can reduce the level of aldehydes such as 3-methylbutyraldehyde during fruit ripening, whereas the short-chain protein encoding $\mathrm{CmADH2}$ cannot; these two $\mathrm{ADH}$ genes work together to influence the production of aromatic compounds (Manriquez et al., 2006). Integrated transcriptome, qRT-PCR and GC-MS analyses revealed that the expression levels of three $A D H$ genes were closely correlated with the content of aromatic compounds during fruit development, suggesting the roles of these three genes in catalyzing aldehydes to alcohols during pear fruit ripening stage (Zeng et al., 2020). We found that PuADH2 shares $\geq 80 \%$ amino acid sequence identity with $A D H$ s from apple, cherry, strawberry, cassava, longan, and grape. Gene Ontology prediction indicated that PuADH2 may involve in oxidation-reduction processes and $\mathrm{PUADH} 2$ acts preferentially as an oxidase, which may catalyze the oxidation of alcohols to aldehydes to aromatic precursors during fruit ripening.

In this study, we showed that the PuADH2 activity was higher on hexanol with $\mathrm{NAD}^{+}$as a cofactor than that on hexanal with NADH as a cofactor. We suggested that PuADH2 be an oxidase, similar to the $A D H$ s from olive and mango (Salas and Sánchez, 1998; Singh et al., 2010). Furthermore, its oxidase activity can be detected regardless of substrate specificity. Grape fruits produce higher levels of $A D H$ with increasing activity in accordance with the ripening period (Tesniere and Verries, 2000). The $A D H$ activity also resembles to the switch from a predominance of aldehydes to alcohols with the ripening of berry (Kalua and Boss, 2009). The pear aroma reportedly consists of $>300$ compounds, including esters, aldehydes, ketones, hydrocarbons, alcohols, and sulfur (Suwanagul and Richardson, 1997). The expression pattern of $\mathrm{PuADH} 2$ was opposite to that observed based on the hexyl ester-volatile content with the ripening of pear fruit (Qin et al., 2017), indicating that PUADH2 may oxidize alcohols to their corresponding aldehydes to be released or further oxidized during ester-volatile biosynthesis.

Many types of ions are capable of inhibiting or activating $A D H$ activity (Zhu et al., 2012). The $P U A D H 2$ activity decreased significantly along with increased $\mathrm{Zn}^{2+}$ concentrations (Figure 6B), which was corresponding with the effects of $\mathrm{Zn}^{2+}$ on $A D H$ from Desulfovibrio gigas (Hensgens et al., 1993). One explanation for this might be that PuADH2 obtains $\mathrm{Zn}^{2+}$ ions from the culture medium of gene expression experiment. Despite dialysis against MES, it was difficult to eliminate $\mathrm{Zn}^{2+}$ from the buffer. The higher the concentration of $\mathrm{Zn}^{2+}$ in the reaction mixture, the more $\mathrm{Zn}^{2+}-\mathrm{NAD}^{+}$complexes are formed (Vallee and Hoch, 1955), resulting in fewer $\mathrm{NAD}^{+}$cofactors available for $\mathrm{PuADH} 2$. We found that $50 \mu \mathrm{M}$ $\mathrm{K}^{+}$had enhanced PuADH2 enzyme activity, which was consistent with the effects observed in Thermus thermophilus (Pennacchio et al., 2008). Furthermore, PuADH2 showed a minimal activity when $\mathrm{Ca}^{2+}$ was present, although an upregulated $A D H$ expression was reported following calcium treatment (Wei et al., 2016).

\section{Conclusion}

In this study, a PuADH2 gene was cloned from P. ussuriensis 'Nanguoli' and expressed in E. coli cells to produce a protein (PuADH2) of 380-amino acids with a predicted molec- ular weight of $46 \mathrm{kDa}$. Biochemical analysis suggested that PuADH2 be an oxidase, no preference for coenzyme factors $\left(\mathrm{NAD}^{+}\right.$and $\left.\mathrm{NADP}^{+}\right)$. Its activity was enhanced by $50 \mu \mathrm{M} \mathrm{K}^{+}$, minimally affected by $\mathrm{Ca}^{2+}$ and $\mathrm{Mn}^{2+}$, but inhibited by $\mathrm{Zn}^{2+}$ under all conditions tested. These results indicated that PUADH2 may involve in aroma biosynthesis by catalyzing alcohol formation, however, its function in pear fruits requires a further study.

\section{Acknowledgments}

This work was supported by the China Agriculture Research System (CARS-28-33) and the National Natural Science Foundation (31301739). We are thankful to Professor Pan Yongbao (Agricultural Research Services, U.S. Department of Agriculture) for the help in polishing language.

\section{References}

Asif, M.H., Dhawan, P., and Nath, P. (2000). A simple procedure for the isolation of high quality RNA from ripening banana fruit. Plant Mol. Biol. Rep. 18(2), 109-115. https://doi.org/10.1007/BF02824018.

Biasini, M., Bienert, S., Waterhouse, A., Arnold, K., Studer, G., Schmidt, T., Kiefer, F., Cassarino, T.G., Bertoni, M., Bordoli, L., Schwede, T., et al. (2014). SWISS-MODEL: modelling protein tertiary and quaternary structure using evolutionary information. Nucleic Acids Res. 42(W1): W252-W258. https://doi.org/10.1093/nar/gku340.

Chase, T. (1999). Alcohol dehydrogenases: Identification and names for gene families. Plant Mol. Biol. Rep. 17(4), 333-350. https://doi. org/10.1023/A:1007620627083.

Chen, X., Fedrizzi, B., Kilmartin, P.A., and Quek, S.Y. (2020). Development of volatile organic compounds and their glycosylated precursors in tamarillo (Solanum betaceum Cav.) during fruit ripening: A prediction of biochemical pathway. Food Chem. 339. https://doi.org/10.1016/j.foodchem.2020.128046.

Elleuche, S., Fodor, K., Heyde, A.V.D., Klippel, B., Wilmanns, M., and Antranikian, G. (2014). Group III alcohol dehydrogenase from Pectobacterium atrosepticum: Insights into enzymatic activity and organization of the metal ion-containing region. Appl. Microbiol. Biotechnol. 98(9), 4041-4051. https://doi.org/10.1007/s00253013-5374-z.

Fan, F., Lorenzen, J.A., and Plapp, B.V. (1991). An aspartate residue in yeast alcohol dehydrogenase I determines the specificity for coenzyme. Biochemistry 30(26), 6397-6401. https://doi. org/10.1021/bi00240a008.

González-Agüero, M., Troncoso, S., Gudenschwager, O., and CamposVargas, R. (2009). Differential expression levels of aroma-related genes during ripening of apricot (Prunus armeniaca L.). Plant Physiol. Biochem. 47(5), 435-440. https://doi.org/10.1016/j. plaphy.2009.01.002.

Hensgens, C.M.H., Vonck, J., Beeumen, J.V., Bruggen, E.F.J.V., and Hansen, T.A. (1993). Purification and characterization of an oxygenlabile, NAD-dependent alcohol dehydrogenase from Desulfovibrio gigas. J. Bacteriol. 175(10), 2859-2863. https://doi.org/10.1128/ jb.175.10.2859-2863.1993.

Jones, P., Binns, D., Chang, H., Fraser, M., Li, W.Z., McAnulla, C., McWilliam, H., Maslen, J., Mitchell, A., Nuka, G., Pesseat, S., Quinn, A.F., Sangrador-Vegas, A., Scheremetjew, M., Yong, S., Lopez, R., and Hunter, S. (2014). InterProScan 5: Genome-scale protein function classification. Bioinformatics 30(9), 1236-1240. https://doi. org/10.1093/bioinformatics/btu031.

Kalua, C.M., and Boss, P.K. (2009). Evolution of volatile compounds during the development of Cabernet Sauvignon grapes (Vitis vinifera L.). J. Agric. Food Chem. 57(9), 3818-3830. https://doi.org/10.1021/ jf803471n. 
Kumar, S., Jain, K.K., Singh, A., Panda, A., and Kuhad, R.C. (2015). Characterization of recombinant pectate lyase refolded from inclusion bodies generated in E. coli BL21 (DE3). Protein Express. Purif. 110, 43-51. https://doi.org/10.1016/j.pep.2014.12.003.

Longhurst, T., Lee, E., Hinde, R., Brady, C., and Speirs, J. (1994). Structure of the tomato $A D H 2$ gene and Adh2 pseudogenes, and a study of Adh2 gene expression in fruit. Plant Mol. Biol. 26(4), 10731084. https://doi.org/10.1007/BF00040690.

Manríquez, D., El-Sharkawy, I., Flores, F.B., EI-Yahyaoui, F., Regad, F., Bouzayen, M., Latche, A., and Pech, J. (2006). Two highly divergent alcohol dehydrogenases of melon exhibit fruit ripening-specific expression and distinct biochemical characteristics. Plant Mol. Biol. 61(4), 675-685. https://doi.org/10.1007/s11103-006-0040-9.

Molina, I., Salles, C., Nicolas, M., and Crouzet, J. (1987). Grape alcohol dehydrogenase. II. Kinetic studies: Mechanism, substrate, and coenzyme specificity. Am. J. Enol. Viticult. 38(1), 60-64.

Pennacchio, A., Pucci, B., Secundo F., Cara, F.L., Rossi, M., and Raia, C.A. (2008). Purification and characterization of a novel recombinant highly enantioselective short-chain NAD (H)-dependent alcohol dehydrogenase from Thermus thermophilus. Appl. Environm. Microb. 74(13), 3949-3958. https://doi.org/10.1128/AEM.00217-08.

Qin, G.H., Tao, S.T., Cao, Y.F., Wu, J.Y., Zhang, H.P., Huang, W.J., and Zhang, S.L. (2012). Evaluation of the volatile profile of 33 Pyrus ussuriensis cultivars by HS-SPME with GC-MS. Food Chem. 134(4), 2367-2382. https://doi.org/10.1016/j.foodchem.2012.04.053.

Qin, G.H., Qi, X.X., Qi, Y.J., Gao, Z.H., Yi, X.K., Pan, H.F., and Xu, Y.L. (2017). Identification and expression patterns of alcohol dehydrogenase genes involving in ester volatile biosynthesis in pear fruit. J. Integr. Agric. 16(8), 1742-1750. https://doi.org/10.1016/ S2095-3119(17)61686-1.

Saitou, N., and Nei, M. (1987). The neighbor-joining method: A new method for reconstructing phylogenetic trees. Mol. Biol. Evol. 4(4), 406-425.

Salas, J.J., and Sánchez, J. (1998). Alcohol dehydrogenases from olive (Olea europaea) fruit. Phytochemistry 48(1), 35-40. https://doi. org/10.1016/S0031-9422(97)01097-2.

Singh, R.K., Sane, V.A., Misra, A., Ali, S., and Nath, P. (2010). Differential expression of the mango alcohol dehydrogenase gene family during ripening. Phytochemistry 71(13), 1485-1494. https://doi. org/10.1016/j.phytochem.2010.05.024.

Singh, R.K., Srivastava, S., Chidley, H.G., Nath, P., and Sane, V.A. (2018). Overexpression of mango alcohol dehydrogenase (MiADH1) mimics hypoxia in transgenic tomato and alters fruit flavor components. Agri Gene 7, 23-33. https://doi.org/10.1016/j.aggene.2017.10.003.

Suwanagul, A., and Richardson, D.G. (1997). Identification of headspace volatile compounds from different pear (Pyrus communis L.) varieties. Acta Hortic. 475, 605-624. https://doi.org/10.17660/ ActaHortic.1998.475.73.

Tesniere, C., and Verries, C. (2000). Molecular cloning and expression of cDNAs encoding alcohol dehydrogenases from Vitis vinifera L. during berry development. Plant Sci. 157(1), 77-88. https://doi. org/10.1016/S0168-9452(00)00274-0.

Thompson, J.D., Higgins, D.G., and Gibson, T.J. (1997). CLUSTAL $\mathrm{X}$ multiple sequence alignment program (Hamburg, Germany: European Molecular Biology Organization).

Vallee, B.L., and Hoch, F.L. (1955). Zinc, a component of yeast alcohol dehydrogenase. Proc. Nat. Acad. Sci. 41(6), 327-338. https://doi. org/10.1073/pnas.41.6.327.

Wei, S.W., Tao, S.T., Qin, G.H., Wang, S.M., Tao, J.H., Wu, J., Wu, J.Y., and Zhang, S.L. (2016). Transcriptome profiling reveals the candidate genes associated with aroma metabolites and emission of pear (Pyrus ussuriensis). Sci. Hortic. 206, 33-42. https://doi.org/10.1016/j.scienta.2016.04.019.
Wu, J., Wang, Z., Shi, Z., Zhang, S., Ming, R., Zhu, S., Khan, M.A., Tao, S., et al. (2013). The genome of the pear (Pyrus bretschneideri Rehd.). Genome Res. 23(2), 396-408. https://doi.org/10.1101/ gr.144311.112.

Yokoyama, S., and Harry, D.E. (1993). Molecular phylogeny and evolutionary rates of alcohol dehydrogenases in vertebrates and plants. Mol. Biol. Evol. 10(6), 1215-1226.

Zeng, W.W., Qiao, X., Li, Q.H., Liu, C.X., Wu, J., Yin, H., and Zhang, S.L. (2020). Genome-wide identification and comparative analysis of the $A D H$ gene family in Chinese white pear (Pyrus bretschneideri) and other Rosaceae species. Genomics 112(5), 3484-3496. https://doi. org/10.1016/j.ygeno.2020.06.031.

Zhang, B., Shen, J.Y., Wei, W.W., Xi, W.P., Xu, C.J., Ferguson, I., and Chen, K. (2010). Expression of genes associated with aroma formation derived from the fatty acid pathway during peach fruit ripening. J. Agric. Food. Chem. 58(10), 6157-6165. https://doi.org/10.1021/ jf100172e.

Zhu, J., Shi, J., and Pan, Z. (2012). Purification and characterization of a hexanol-degrading enzyme extracted from apple. J. Agric. Food. Chem. 60(12), 3246-3252. https://doi.org/10.1021/jf204548r.

Received: Sep. 4, 2020

Accepted: Jul. 21, 2021

Addresses of authors:

Qin Gaihua ${ }^{1,2, \dagger}$, Liu Chunyan ${ }^{1,2, \dagger}$, Gao Zhenghui ${ }^{1,2}$, Li Jiyu ${ }^{1,2}$, Qi Yongjie ${ }^{1,2}$, Pan Haifa ${ }^{1}$, Yi Xingkai ${ }^{1}$ and Xu Yiliu ${ }^{1,2, *}$

${ }^{1}$ Key Laboratory of Horticultural Crop Genetic Improvement and Eco-physiology of Anhui Province, Institute of Horticulture Research, Anhui Academy of Agricultural Sciences, Hefei 230031, China

${ }^{2}$ Key Laboratory of Fruit Quality and Developmental Biology, Anhui Academy of Agricultural Sciences,

Hefei 230031, China

† Equal contributors

*Corresponding author; E-mail: yiliuxu@163.com 
